\title{
MHD seismology of coronal loops using the period and damping of quasi-mode kink oscillations
}

\author{
I. Arregui ${ }^{1}$, J. Andries ${ }^{2, \star}$, T. Van Doorsselaere ${ }^{2}$, M. Goossens ${ }^{2}$, and S. Poedts ${ }^{2}$ \\ 1 Departament de Física, Universitat de les Illes Balears, 07122 Palma de Mallorca, Spain \\ e-mail: inigo.arregui@uib.es \\ 2 Centrum voor Plasma Astrofysica, KU Leuven, Celestijnenlaan 200B, 3001 Heverlee, Belgium \\ e-mail: [Jesse. Andries; tomvd; Marcel.Goossens; Stefaan.Poedts]@wis.kuleuven.be
}

Received 20 June 2006 / Accepted 11 November 2006

ABSTRACT

\begin{abstract}
Aims. We combine the magnetohydrodynamic (MHD) theory of resonantly damped quasi-mode kink oscillations with observational estimates of the period and damping of transverse coronal loop oscillations to extract information on physical parameters in oscillating loops.

Methods. A numerical study of the quasi-mode period and damping, in one-dimensional fully non-uniform flux tubes, is used to obtain equilibrium models that reproduce the observed periods and damping rates. This scheme is applied to 11 loop oscillation events.

Results. When only the damping rate is used, the valid equilibrium models form a one-dimensional solution curve in the twodimensional parameter space (density contrast, transverse inhomogeneity length-scale). Lower limits to the transverse inhomogeneity are obtained in the limit of high contrast loops. When both the period and the damping rate are used, the equilibrium Alfvén speed (or Alfvén travel time) comes into play. The valid equilibrium models then form a one-dimensional solution curve in the three-dimensional parameter space (density contrast, transverse inhomogeneity length-scale, Alfvén speed or Alfvén travel time). The projection of these solutions onto the Alfvén speed axis is found to be constrained to a rather limited interval. Upper limits to the internal Alfvén speed are derived for 9 of the 11 analysed events.
\end{abstract}

Key words. magnetohydrodynamics (MHD) - Sun: corona - Sun: magnetic fields - Sun: oscillations

\section{Introduction}

The idea of coronal seismology, the determination of unknown physical parameters of the solar corona by a comparison of observed with theoretical properties of waves and oscillations in magnetic plasma configurations, goes back to Uchida (1970) and Roberts et al. (1984). The recent clear observational evidence of waves and oscillations in several coronal magnetic structures, provided by instruments on board SOHO and TRACE and the refinement of theoretical models, has given a strong impetus to coronal seismology. For example, the interpretation of transverse coronal loop oscillations in terms of the global MHD fast kink eigenmode of a uniform flux tube, in its fundamental harmonic, has been used to estimate the coronal magnetic field strength and transport coefficients (Nakariakov et al. 1999; Nakariakov \& Ofman 2001). The detection of double mode kink oscillations in several loops in a coronal arcade, by Verwichte et al. (2004), has been used by Andries et al. (2005a, based on calculations of Andries et al. 2005b) to determine the density stratification in those loops. More recently, a model of vertically polarised fast magnetoacoustic waves in curved coronal loops has been used to apply the method of coronal seismology to observations of transverse loop oscillations (Verwichte et al. 2006).

An important property of the observed transverse loop oscillations is their rapid damping, with an exponential decay time of only a few periods. The physical nature of the damping

* Postdoctoral Fellow of the National Fund for Scientific ResearchFlanders (Belgium) (FWO-Vlaanderen). mechanism(s) is unknown and still under intensive discussion. Several mechanisms are currently under study, such as non-ideal effects; lateral wave leakage due to the curvature of the loops (Roberts 2000); mechanisms based on the topology of magnetic field lines (Schrijver \& Brown 2000); foot-point leakage through the chromospheric density gradient (De Pontieu et al. 2001; Ofman 2002); phase mixing of Alfvén waves (Heyvaerts \& Priest 1983; Ofman \& Aschwanden 2002); and resonant absorption of quasi-mode kink oscillations (Hollweg \& Yang 1988; Ruderman \& Roberts 2002; Goossens et al. 2002, 2006). A first analysis of the damping rates due to different mechanisms indicates that the latter seems to be the most relevant (Ruderman 2005), although other possibilities cannot be excluded.

This paper is concerned with the damping of coronal loop oscillations by resonant absorption of quasi-mode kink oscillations and the combination of theory and observations to extract information about the physical conditions inside the coronal loops. Recently, theoretical models for the computation of the damping of quasi-mode kink oscillations have been refined, by including fully non-uniform radial and longitudinal density profiles (Van Doorsselaere et al. 2004; Arregui et al. 2005). These computations allow a better comparison between observations and theory, thus improving our prospects for coronal seismology. In the present paper, we use the parametric numerical study by Van Doorsselaere et al. (2004), for 1D fully non-uniform loops together with observed period and damping rates, for a set of 11 loop oscillation events, to derive the equilibrium models that reproduce observations. The assumption of a particular damping mechanism defines a particular relation between the equilibrium 
parameters of seismic interest that reproduces the observed oscillatory properties. Thus, the combination of theory and observations can also be used to test the theory. For this reason, we do not make any a priori assumptions on any of the parameters of interest. All the assumptions are contained in the modelling, but not in the comparison with observations.

The layout of the paper is as follows. In Sect. 2 we describe the physical model and the numerical solutions for resonantly damped fast MHD kink quasi-modes. Also, the physical parameters and observed quantities that are relevant from the seismological point of view are described. Section 3 presents our analysis and results. First, we only use the damping rate to derive equilibrium models that can reproduce the observations. Then, observational estimates of the period and damping rate of 11 loop oscillation events are used to obtain the valid equilibrium models. Finally, in Sect. 4, a summary and a discussion are presented.

\section{Physical model, linear MHD waves and numerical solutions}

A coronal loop is modelled as a gravity-free, straight, cylindrically symmetric flux tube. In a system of cylindrical coordinates $(r, \varphi, z)$ with the $z$-direction coinciding with the axis of the cylinder (loop), the magnetic field is pointing in the $z$-direction. Since the plasma pressure is much smaller than the magnetic pressure in the corona, the neglect of the plasma pressure yields a good approximation. As the magnetic field is straight in our loop model, this classic $\beta=0$ approximation implies that the magnetic field is uniform, $\boldsymbol{B}=B \hat{\boldsymbol{e}}_{z}$, and also that the density profile can be chosen arbitrarily. We consider a one-dimensional variation of plasma density, $\rho(r)$, and adopt a sinusoidal non-uniform profile which continuously connects the constant internal density, $\rho_{\mathrm{i}}$, to the constant external density, $\rho_{\mathrm{e}}$, in a transitional layer of length $l / R$ (normalised to the radius of the loop) (see Eq. (15) in Van Doorsselaere et al. 2004).

The equilibrium configuration is then perturbed in order to study small amplitude oscillations of the system. We assume a dependence of the perturbed quantities of the form $\exp (\mathrm{i}(m \varphi+$ $\left.k_{z} z-\omega t\right)$ ), with $m$ and $k_{z}$ the azimuthal and longitudinal wavenumbers and $\omega=\omega_{R}+\mathrm{i} \omega_{I}$ the complex frequency. Only perturbations with $m=1$ displace the axis of the tube and are called kink-modes. The modelling of the photospheric line-tying leads to a longitudinal wavenumber, $k_{z}=n \pi / L$, with $n$ an integer and $L$ the loop length. Then, the $n=1$ case represents the observed fundamental mode with no nodes in the $z$-direction. In the presence of a non-uniform layer, no pure magneto-sonic modes exist and kink modes are always damped quasi-modes, due to their coupling to Alfvén waves. For these modes, the real part of the frequency corresponds to the oscillation period, $P=2 \pi / \omega_{\mathrm{R}}$, while the damping time is related to the negative imaginary part of the frequency, $\tau_{\mathrm{d}}=-1 / \omega_{I}$.

Numerical eigenmode computations for these 1D fully non-uniform equilibrium models have been carried out by Van Doorsselaere et al. (2004), using the LEDA code (Van der Linden 1991). In this model there are basically four independent quantities. A parametric numerical study of the fast MHD kink eigenmode period and damping rate was performed for a wide range of values for several loop parameters, such as the inverse aspect ratio of the loop $(\varepsilon=\pi R / L)$, the thickness of the inhomogeneous layer $(l / R)$ and the density contrast $\left(\zeta=\rho_{\mathrm{i}} / \rho_{\mathrm{e}}\right)$. The results of those parametric studies give the dependence of the period and damping time of the fundamental fast kink eigenmode in the following form

$$
\begin{aligned}
\frac{P}{\tau_{\mathrm{Ai}}} & =f_{1}(\varepsilon, \zeta, l / R), \\
\frac{\tau_{\mathrm{d}}}{\tau_{\mathrm{Ai}}} & =f_{2}(\varepsilon, \zeta, l / R), \\
\frac{P}{\tau_{\mathrm{d}}} & =f_{3}(\varepsilon, \zeta, l / R) .
\end{aligned}
$$

Here time is normalised with respect to the internal Alfvén travel time $\tau_{\mathrm{Ai}}=L / V_{\mathrm{Ai}}$, with $V_{\mathrm{Ai}}=B^{2} / \sqrt{\rho_{\mathrm{i}}}$ the internal Alfvén speed. Note that, in Eqs. (1)-(3), only two of the three functions are independent.

From an observational point of view, some quantities occurring in Eqs. (1)-(3) are easier to estimate than others. For each loop oscillation event the loop length $(L)$, radius $(R)$, period $(P)$ and decay time $\left(\tau_{\mathrm{d}}\right)$ are in principle available from observations, although several assumptions have to be made in order to derive these quantities. For example, the length of the loop is usually estimated by measuring the distance between the foot-points and assuming a semi-circular shape for the loop. The estimation of the radius of the loop is also subject to considerable modelling. Measurements of periods and damping times also have their corresponding errors. Due to line of sight integration it is very hard (if not impossible) to determine the cross sectional profile of the density (here represented by the density contrast $\zeta$, and the inhomogeneity length scale $l / R$ ). The relations (1)-(3) can thus be used to try to infer values for the parameters which are inaccessible to direct measurement, based on the values of the parameters that can be measured with much more confidence. From such a seismological point of view it is important to remark right from the start how the relations (1)-(3) depend on the different parameters. First off all it must be noted that the inverse aspect ratio $\varepsilon$ of the loops under consideration is very small. In that range the functions $f_{i}$ in the right hand sides of Eqs. (1)-(3) are largely independent of the precise value of the inverse aspect ratio. In the calculations to follow, the observed value of the inverse aspect ratio is used but the results are robust with respect to errors in the measurement of that parameter. Thus we basically only retain $l / R$ and $\zeta$ as parameters in the right hand sides. Secondly, the Alfvén speed and the length of the loop only enter through the Alfvén travel time, which in turn only enters as the appropriate time normalisation. In other words, a seismological comparison of the observed damping times and periods with the nondimensional Eqs. (1) and (2) yields the Alfvén travel time as a basic parameter. We can thus summarise the problem as follows, given the observed values of the periods and damping rates, we would like to determine the values of the inhomogeneity length scale $l / R$, the density contrast $\zeta$, and the Alfvén travel time $\tau_{\mathrm{Ai}}$. This means that, with the current model and the available observations, any method for obtaining a seismic determination of the unknown parameters using the observed period and damping of loop oscillations can only yield a 1D solution space containing the equilibrium models that reproduce the observations, unless some additional assumption is made. In the next section, the solution space is obtained and its properties are studied.

\section{Analysis and results}

In this section we use the theory of resonantly damped quasimode kink oscillations to derive valid equilibrium models that reproduce the observed periods and damping times of transverse loop oscillations. The time unit, and hence the Alfvén speed, can be eliminated using Eq. (3) and studying $P$ and $\tau_{\mathrm{d}}$ relative 
to each other. The thin tube and thin boundary (TTTB) approximation furthermore eliminates the $\varepsilon$-dependence and simplifies the $l / R$-dependence. This non-dimensional analysis is described in Sect. 3.1. In Sect. 3.2 we reintroduce the time dimension and describe the analysis using both the period and the damping rate of loop oscillations. As we still have two relations and three parameters the solution for the valid equilibrium models that reproduce observations is still a 1D solution space, in the 3D parameter space $\left(\zeta, l / R, \tau_{\mathrm{Ai}}\left(P, \tau_{\mathrm{d}}\right)\right)$. When one quantity is fixed, the other two can be computed. This scheme is applied to a set of 11 observed loop oscillation events.

\subsection{Seismology using the damping rate of loop oscillations}

We first consider resonantly damped eigenmodes, in onedimensional flux tube models, under the thin tube $(R / L \ll 1)$ and thin boundary $(l / R \ll 1)$ approximations. These approximations allow us to write down an analytical expression for the damping rate of the kink-mode of the form (Hollweg \& Yang 1988; Goossens et al. 1992; Tirry \& Goossens 1996; Ruderman \& Roberts 2002)

$\frac{\mathrm{P}}{\tau_{\mathrm{d}}}=\delta\left(\frac{l}{R}\right)\left(\frac{\zeta-1}{\zeta+1}\right)$,

where $\delta$ is a parameter that depends on the density profile at the non-uniform layer (e.g. $\pi^{2} / 4$ for a linear ramp, $\pi / 2$ for a sinusoidal profile, etc.). The damping formula (Eq. (4)) establishes a relation between the damping rate on one side, and two equilibrium physical parameters, $\zeta$ and $l / R$, which are difficult to obtain from observations, on the other side. For a given damping rate, this equation links the possible values of these two equilibrium quantities, under the resonant absorption mechanism and, hence, completely characterises the theoretical predictions for the damping rate under this particular damping mechanism. For a given form of the non-uniform radial profile (a given $\delta$ ), Eq. (4) defines in the $(\zeta, l / R)$-plane a one-parametric family of curves which may be called "iso-damping rate" curves. Given an observational value of $P / \tau_{\mathrm{d}}$, the corresponding iso-damping rate curve can be drawn in the $(\zeta, l / R)$-plane. This curve then indicates all possible combinations of equilibrium configurations which can reproduce the observed damping rate.

Three of these curves are shown in Fig. 1, for three different values of the damping rate. Each of these curves specifies a set of points on the $(\zeta, l / R)$-plane that correspond to different loop equilibrium models. As can be appreciated, a particular value for the observed damping rate can be recovered by an infinite number of equilibrium models ranging from models with thin non-uniform transitional layers (low inhomogeneous loops) and large density contrast to models with thicker non-uniform layers (larger inhomogeneous loops) and lower density contrast. When we increase the damping rate, the curves are shifted towards the right top corner in the parameter space and valid equilibrium models have both larger density contrasts and longer inhomogeneity length-scales. The grey-shaded area represents equilibrium models for which the damping mechanism predicts damping times shorter than the oscillatory period. This region represents a problem from an observational point of view, since oscillations would be, in that case, difficult to observe.

The analysis using the damping rate provides us with one relation between two quantities, $\zeta$ and $l / R$. When one of those quantities is fixed, the other one can be computed. Figure 1 shows that a given measurement error on $\zeta$ would produce a smaller uncertainty on the determination of $l / R$ for large density contrast loops than for very low contrast ones, due to the

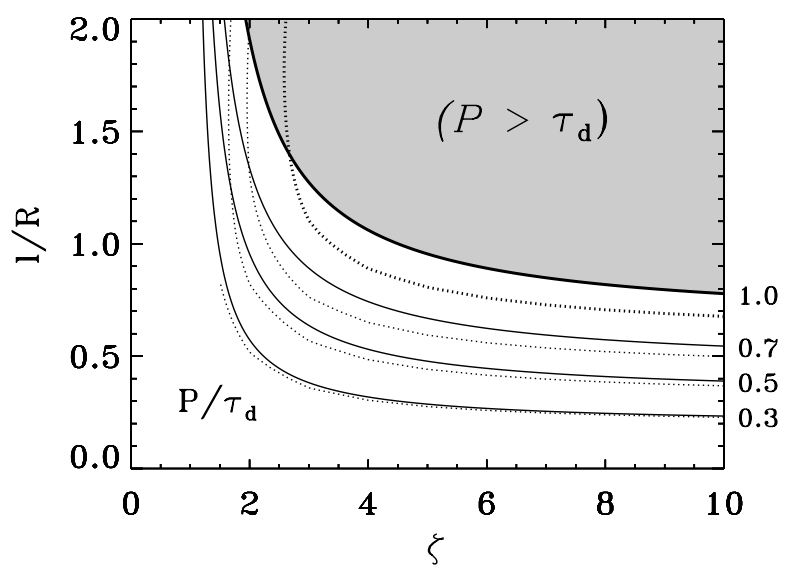

Fig. 1. Curves of constant damping rate for three different values of $P / \tau_{\mathrm{d}}=-2 \pi\left(\omega_{I} / \omega_{R}\right)=0.3,0.5$ and 0.7 . The continuous lines correspond to curves obtained using Eq. (4), with a linear non-uniform profile in the transitional layer. Dotted lines correspond to curves obtained using numerical solutions for fully non-uniform loops by Van Doorsselaere et al. (2004). They all show the location of the different equilibrium models, on the $(\zeta, l / R)$-plane, that reproduce those particular damping rates. Also shown, in thicker dotted and full lines, are the curves corresponding to $P=\tau_{\mathrm{d}}$, above which (grey-shaded area) the period is larger than the damping time.

flattening of the iso-damping rate curves for large density contrasts. Equation (4) was used by Goossens et al. (2002) to show that damping by resonant absorption of quasi-mode kink oscillations gives a consistent explanation of the rapid decay, if the inhomogeneity scale-length is allowed to vary from loop to loop. Goossens et al. (2002) took $\zeta=10$ and found values for $l / R$ in between 0.16 and 0.49 for a selection of 11 loop oscillation events. Figure 1 confirms these results, and indicates that, depending on the value of the damping rate, $l / R$ has to be roughly between 0.25 and 0.8 for a fixed density contrast of $\zeta=10$. Equation (4) predicts a minimum value of $l / R$ which, under the TTTB approximation, can be written as $(l / R)_{\min }=l / R(\zeta \rightarrow$ $\infty)=\delta^{-1} P / \tau_{\mathrm{d}}$. This allows us to obtain an estimate of the lower limit of the transverse inhomogeneity length-scale in the limit of high contrast loops. Lower density contrasts lead to larger values of the inhomogeneity length-scale and, consequently, the TTTB approximation is not valid any longer.

For realistic values of $l / R$, we can obtain similar curves of constant damping rate by using the numerical computations, given by Eq. (3), instead of the damping formula (Eq. (4)). We take $\varepsilon=0.06$, a typical value for coronal loops, and plot curves of constant damping rate, for the same values of the damping rate as before (see Fig. 1). The shape of the curves on the $(\zeta$, $l / R)$-plane turns out to be similar, but the analysis is now consistent with the fact that highly non-uniform radial profiles are included. The curves point now to slightly different equilibrium models and the two sets of curves do not agree. This is due to the differences in damping rate between the TTTB results and the fully non-uniform results (Van Doorsselaere et al. 2004). Now the same damping rate is attained for slightly shifted equilibrium configurations. As shown by Van Doorsselaere et al. (2004), these differences increase for large inhomogeneity length-scales and high damping rates. Note that for relatively low damping rates $\left(P / \tau_{\mathrm{d}}=0.3\right.$, for example), the curves do not cover the full parameter space. In this case, the limitation originates from the minimum density contrast considered in the numerical simulations $(\zeta=1.5)$. 
Table 1. Summary of physical and geometrical properties of the 11 loop oscillation events selected by Ofman \& Aschwanden (2002); Goossens et al. (2002) (left hand-side) and computed constraints for the valid equilibrium models (right hand-side), in the range of density contrasts $\zeta \in$ [1.5-20]. Events marked with an asterisk do not exhibit physical constraints on the upper values of $l / R$ and $V_{\mathrm{Ai}}$ since these constraints originate in these cases from the limitations used in the numerical solutions $(\zeta \geq 1.5)$.

\begin{tabular}{ccccccc|ccc}
\hline \hline Loop & $R\left(10^{6} \mathrm{~m}\right)$ & $L\left(10^{8} \mathrm{~m}\right)$ & $\varepsilon=\pi R / L$ & $P(\mathrm{~s})$ & $\tau_{\mathrm{d}}(\mathrm{s})$ & $P / \tau_{\mathrm{d}}$ & $l / R$ & $\tau_{\mathrm{Ai}}(\mathrm{s})$ & $V_{\mathrm{Ai}}=L / \tau_{\mathrm{Ai}}\left(\mathrm{km} \mathrm{s}^{-1}\right)$ \\
\hline 1 & 3.60 & 1.68 & 0.067 & 261 & 870 & 0.30 & $0.20-0.83 \star$ & $155-190$ & $880-10800^{\star}$ \\
2 & 3.35 & 0.72 & 0.146 & 265 & 300 & 0.88 & $0.58-2.0$ & $378-423$ & $170-190$ \\
3 & 4.15 & 1.74 & 0.075 & 316 & 500 & 0.63 & $0.41-2.0$ & $225-259$ & $670-770$ \\
4 & 3.95 & 2.04 & 0.061 & 277 & 400 & 0.69 & $0.45-2.0$ & $163-183$ & $1110-1250$ \\
5 & 3.65 & 1.62 & 0.071 & 272 & 849 & 0.32 & $0.22-0.90 \star$ & $170-210$ & $770-950^{\star}$ \\
6 & 8.40 & 3.90 & 0.068 & 522 & 1200 & 0.44 & $0.30-2.0$ & $327-386$ & $1010-1190$ \\
7 & 3.50 & 2.58 & 0.043 & 435 & 600 & 0.73 & $0.47-2.0$ & $181-203$ & $1270-1420$ \\
8 & 3.15 & 1.66 & 0.059 & 143 & 200 & 0.72 & $0.46-2.0$ & $85-93$ & $1780-1950$ \\
9 & 4.60 & 4.06 & 0.036 & 423 & 800 & 0.53 & $0.35-2.0$ & $140-165$ & $2460-2900$ \\
10 & 3.45 & 1.92 & 0.056 & 185 & 200 & 0.93 & $0.57-2.0$ & $105-113$ & $1690-1830$ \\
11 & 7.90 & 1.46 & 0.169 & 396 & 400 & 0.98 & $0.62-2.0$ & $663-730$ & $200-220$ \\
\hline
\end{tabular}

In conclusion, the use of the damping rate of loop oscillations alone allows us to only establish a one-to-one relation between two physical parameters of interest. As Fig. 1 shows, there are an infinite number of different equilibrium models, with different values of $\zeta$ and $l / R$, that yield the same observational value of the damping rate and it is impossible to discriminate between them. If one of the parameters is fixed the other can be obtained. An attempt to do so was published by Aschwanden et al. (2003).

\subsection{Seismology using the period and damping rate of loop oscillations}

Both the period and the damping rate provide us with important and largely independent sources of information about the physical conditions in oscillating coronal loops. The analysis in this section combines the observed period and damping rate for the computation of valid equilibrium models that reproduce observations. Here, we introduce a new observable, the period, but also a new parameter, the Alfvén travel time. As we do not make any additional assumption on any equilibrium parameter, again a $1 \mathrm{D}$ solution curve will be obtained in the new $\left(\zeta, l / R, \tau_{\mathrm{Ai}}\right)$ parameter space.

Since the first TRACE observation of damped transverse loop oscillations near the end of the last century (Aschwanden et al. 1999; Nakariakov et al. 1999) several similar events have been observed. The statistics and derived physical properties of these events can be found in Aschwanden et al. (2002) and Schrijver et al. (2002). A selection of 11 of those events was used by Ofman \& Aschwanden (2002) and Goossens et al. (2002) in their analysis of phase mixing and resonant absorption as damping mechanisms. Here, the same selection of loop oscillation events is considered. The physical and geometrical properties of interest for these loops are collected in the left-hand side of Table 1.

From the computational side we use the output from the parametric study by Van Doorsselaere et al. (2004), expressed by Eqs. (1) and (3). Values for the inverse aspect ratio $\varepsilon$ of the loops are obtained from the estimates of length and radius in Table 1, but as stated before the results are robust with respect to that parameter. In order to incorporate the time unit to our analysis, a three-dimensional data-cube is constructed by considering a range of values for the Alfvén travel time. To determine the valid equilibrium models for all loop oscillation events in Table 1, a corresponding range of $V_{\mathrm{Ai}}$ from 0 to $3000 \mathrm{~km} \mathrm{~s}^{-1}$ turns out to be sufficient. We have used a sampling of $\Delta V_{\mathrm{Ai}}=10 \mathrm{~km} \mathrm{~s}^{-1}$. Finally, we thus end up with two three-dimensional data cubes

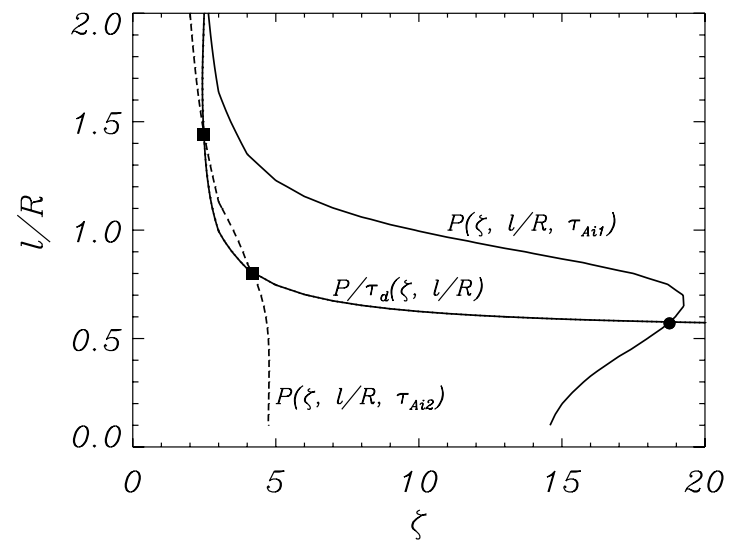

Fig. 2. Curves of constant period ( $P=185 \mathrm{~s}$ ) and constant damping rate $\left(P / \tau_{\mathrm{d}}=0.93\right)$ on the $(\zeta, l / R)$-plane corresponding to loop oscillation event \#10, for two different values of the internal Alfvén travel time; $\tau_{\mathrm{Ai} 1}=113 \mathrm{~s}$ (solid lines) and $\tau_{\mathrm{Ai} 2}=106 \mathrm{~s}$ (dashed lines). The filled circle indicates the only valid equilibrium $(\zeta \simeq 18.8, l / R \simeq 0.57)$ that reproduces the observed wave characteristics for $\tau_{\mathrm{Ai} 1}$. When $\tau_{\mathrm{Ai}}=\tau_{\mathrm{Ai} 2}$, two different equilibrium models (filled squares) are possible; $(\zeta \simeq 4.2$, $l / R \simeq 0.8)$ and $(\zeta \simeq 2.48, l / R \simeq 1.44)$.

of the form, $P=P\left(\zeta, l / R, \tau_{\mathrm{Ai}}\right)$ and $P / \tau_{\mathrm{d}}=P / \tau_{\mathrm{d}}(\zeta, l / R)$ for each loop oscillation event. These data-cubes can now be combined with the observed periods and damping rates by requiring that

$$
\begin{aligned}
& P\left(\zeta, l / R, \tau_{\mathrm{Ai}}\right)=\tau_{\mathrm{Ai}} f_{1}(\zeta, l / R)=P_{\mathrm{obs}}, \\
& \frac{P}{\tau_{\mathrm{d}}}(\zeta, l / R)=\left(\frac{P}{\tau_{\mathrm{d}}}\right)_{\mathrm{obs}} .
\end{aligned}
$$

In this way, we obtain the equilibrium models, $\left\{\zeta, l / R, \tau_{\mathrm{Ai}}\right\}$ that reproduce the observed periods and damping rates $\left(P_{\mathrm{obs}}\right.$ and $\left.\left(P / \tau_{\mathrm{d}}\right)_{\mathrm{obs}}\right)$, for each oscillation event.

We first consider loop oscillation event \#10 from Table 1. By using the observed period and damping rate, curves of constant period and constant damping rate can be drawn in the $(\zeta, l / R)$ plane, for given values of the internal Alfvén travel time. For two different values, these curves are depicted in Fig. 2. Because the period depends on the Alfvén travel time, two curves are shown, pointing at different locations in the parameter space. On the other hand, curves of constant damping rate are independent of the internal Alfvén travel time and the curves for both Alfvén travel times overlap. The valid equilibrium models, for which the observed period and damping rate are reproduced, are given by the crossing points of both kind of curves, with the 
same Alfvén travel time. They all have to belong to the curve of constant damping rate. Hence, this curve gives the valid equilibrium models for all Alfvén travel times. For one of the selected Alfvén travel times $\left(\tau_{\mathrm{Ai}}=\tau_{\mathrm{Ai} 1}\right)$ a single equilibrium model, with fixed values of $\zeta$ and $l / R$, is obtained. If we choose another value for the Alfvén travel time the valid equilibrium model is changed (and vice versa, another crossing point requires another value of the Alfvén travel time). Moreover, for $\tau_{\mathrm{Ai}}=\tau_{\mathrm{Ai} 2}$, two different equilibrium models with equal Alfvén travel time, but different density contrast and inhomogeneity length-scale, are obtained, which can equally well explain the observed period and damping rate.

When we vary $\tau_{\mathrm{Ai}}$, curves of constant period appear first from the right top corner and are then shifted, for decreasing Alfvén travel time, towards the left bottom corner in Fig. 2. This means that valid equilibrium models, in our parameter space, can only exist for a given range of Alfvén travel times. We obtain this set of valid equilibrium models by collecting the successive crossing points along the damping rate curve in Fig. 2, for each allowed Alfvén travel time. These crossing points are obtained numerically by finding the location at which the difference between the two kind of curves changes sign. They form a $1 \mathrm{D}$ solution space in the three-dimensional parameter space $(\zeta$, $\left.l / R, \tau_{\mathrm{Ai}}\right)$. This $1 \mathrm{D}$ solution curve is the result of the intersection of the two surfaces defined by Eqs. (5) and (6) and may easily be obtained by computing the successive crossings of curves like the ones shown in Fig. 2, for the complete assumed range of Alfvén speeds.

Figure 3 displays the $1 \mathrm{D}$ solution spaces in the threedimensional parameter space obtained for loop oscillation events \#5 (Fig. 3a) and \#10 (Fig. 3b). Also shown are the projections of the solution curves onto the different two-dimensional parameter planes. For each event, the projection of the curve onto the horizontal plane is the curve of constant damping rate. As can be appreciated, the values of the Alfvén travel time for valid equilibrium models in our parameter regime are constrained to a rather limited interval. The largest values correspond to equilibrium models with large density contrasts and relatively low inhomogeneity length-scales. Then, moving along the 1D solution curve (and along the damping rate curve in the horizontal plane), valid equilibrium models have lower Alfvén travel time. In both events, the upper limit for the Alfvén travel time is obtained for the largest density contrast. As such, this is not a true lower limit, since the density contrast could be larger. However, the flattening of the 1D solution curve and the increase in the separation of equilibrium models towards larger density contrasts suggest that the Alfvén travel time will not vary significantly when the density contrast is allowed to increase past its current limit. Also, the projection of the curve onto the $(\zeta, l / R)$-plane allows us to obtain an estimate for the lowest value of $l / R$.

In the case of loop oscillation event \#5, the lowest value for the Alfvén travel time $\left(\tau_{\mathrm{Ai}}=170 \mathrm{~s}\right)$ is obtained at $\zeta=1.5$, which coincides with the minimum density contrast considered in the numerical solutions. Thus, the obtained lowest limit for the Alfvén travel time is actually restricted by the limitations on the numerical data and does not constitute a physical upper limit. However, we cannot go much further if we want to have a real loop with $\zeta \geq 1$. For loop oscillation event \#10, the lowest value of the Alfvén travel time for valid equilibrium models $\left(\tau_{\mathrm{Ai}}=\right.$ $105 \mathrm{~s}$ ) is obtained for a larger contrast than the minimum value available from numerical simulations. This allows us to obtain a physical lower limit on $\tau_{\mathrm{Ai}}$. Although we did not make any a priori assumptions for any of the equilibrium parameters, we can obtain a physical lower limit to the internal Alfvén travel time.
After the minimum Alfvén travel time is reached the curve bends up again resulting in a constrained range for the possible values of $\tau_{\mathrm{Ai}}$.

The above described procedure has been applied to the set of 11 loop oscillation events presented in Table 1 . This table shows the lower limits to the inhomogeneity length-scale and the constraints on the Alfvén travel time that have been obtained, within the range of assumed density contrasts $\zeta \in[1.5-20]$. By additionally using the measured value for the loop length $L$ this also determines the internal Alfvén speed which is also displayed in Table 1 . In 9 of the 11 cases, an upper limit to the internal Alfvén speed has been found. The other 2 events correspond to cases in which we are limited by our parameter regime. Because of this limitation, the latter cases also exhibit an upper limit to the inhomogeneity length-scale which is not the one imposed by the modelling $(l / R=2$, for fully non-uniform loops). As for the Alfvén speed, this is not a physical upper limit. When the lower boundary on $\zeta$ would be decreased, higher values for the inhomogeneity length-scales will be allowed.

The values for the internal Alfvén speed obtained for loops \#2 and \#11 are particularly striking (see Table 1). These low values for the Alfvén speed closely correspond to a high value for the inverse aspect ratio $\varepsilon$.

Now let us appreciate these results. The Alfvén speed has been determined based on the observed period of loop oscillations before (Nakariakov \& Ofman 2001). However in those papers the analysis was based on the period only, using the expression for the kink speed in the thin tube limit and in addition assuming a specific value for the density contrast. An attempt for direct measurements of the density contrast (Aschwanden et al. 2003) suggested that the density contrast might be a lot smaller. In fact in that range, the formula for the kink speed becomes very sensitive to the density contrast. The uncertainty on the density contrast thus implies that the Alfvén speed can be about $40 \%$ larger than estimated by those authors. Our analysis, however, based on a more complicated model with less simplifying assumptions and making use of both observed periods and damping times (under the assumption of damping by resonant absorption) turns out to be much more robust with respect to determining the Alfvén speed.

So far we have adopted the rather unrealistic view that the observed period and damping times are without measurement errors. We have repeated the procedure by considering uncertainties of $40 \%$ and $60 \%$ on the period and damping time respectively (Aschwanden et al. 2002). In this way, for loop oscillation event \# 10, for example, the period and damping times would be $P=185 \pm 74 \mathrm{~s}$ and $\tau_{\mathrm{d}}=200 \pm 120 \mathrm{~s}$, respectively. By considering the upper value for the period $(P=259 \mathrm{~s})$ with different values of $\tau_{\mathrm{d}}$, the Alfvén speed can be estimated to be in the range $V_{\mathrm{Ai}} \in(1200-1340) \mathrm{km} \mathrm{s}^{-1}$ (instead of the 1690$1830 \mathrm{~km} \mathrm{~s}^{-1}$ for the case without errors). By considering the value for the period used before $(P=185 \mathrm{~s})$ together with $\tau_{\mathrm{d}}$ in the range $\tau_{\mathrm{d}} \in(80-320 \mathrm{~s}), V_{\mathrm{Ai}}$ can be estimated to be in the range $V_{\mathrm{Ai}} \in(1690-1960) \mathrm{km} \mathrm{s}^{-1}$. The largest differences occur for the smaller value of $P=111 \mathrm{~s}$ with different possibilities for $\tau_{\mathrm{d}}$. In this case, the range for $V_{\mathrm{Ai}}$ is $2650-2870 \mathrm{~km} \mathrm{~s}^{-1}$. These high values for the Alfvén speed are caused by the extremely short period.

\section{Summary and conclusions}

This paper reports on how both the period and the damping time of transverse coronal loop oscillations can be used to obtain information on the physical conditions in oscillating loops. We 

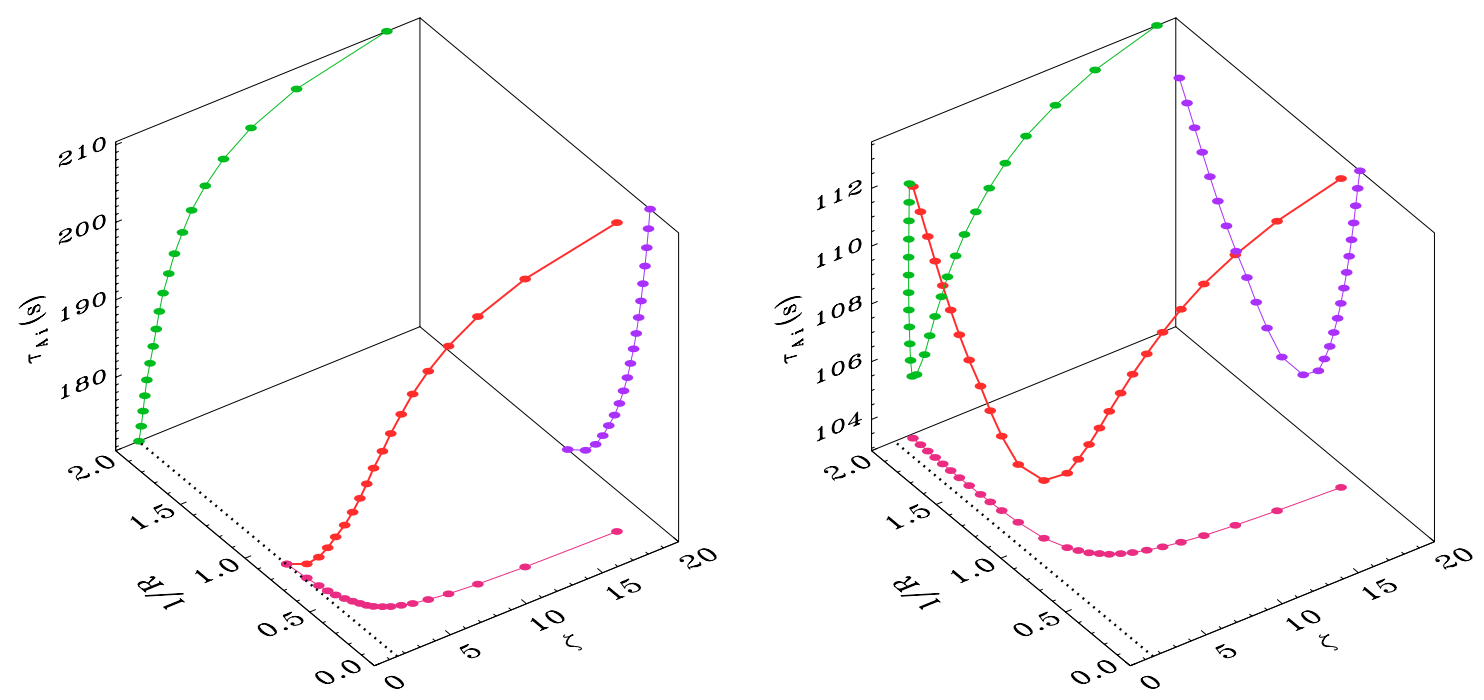

a

Fig. 3. Three-dimensional view of the 1D solution curves representing the valid equilibrium models that reproduce observed periods and damping rates in the parameter space $\left(\zeta, l / R, \tau_{\mathrm{Ai}}\right)$. The solutions are also projected onto the different two-dimensional parameter planes. Circles are representative of the sampling on the internal Alfvén speed, $10 \mathrm{~km} \mathrm{~s}^{-1}$, used for the computation of the curves. The dotted line in the horizontal plane represents the minimum density contrast $(\zeta=1.5)$ considered in the numerical solutions. a) Loop oscillation event \#5 and b) loop oscillation event \#10.

have adopted resonant absorption of quasi-mode kink oscillations as the mechanism responsible for the damping of loop oscillations. Observed values of the period and damping rate have been used together with the results of a parametric numerical study of the complex frequency of quasi-mode kink oscillations in 1D fully non-uniform equilibrium models. The analysis described in this paper allowed the individual study of loop oscillation events and took into account the particular physical and geometrical parameters for each case.

When we only use the damping rate of loop oscillations, the assumed damping mechanism provides us with a relation between the observable damping rate and the density contrast and radial inhomogeneity length-scale, that are difficult to obtain from observational data. This relation, given by the damping rate curve defines a $1 \mathrm{D}$ solution space of different equilibrium configurations which can reproduce the observed damping rate. When only using the damping rate, we have one observable which determines a relationship between two equilibrium parameters. When one of the free parameters is estimated, the other free parameter can be deduced.

As a next step, we have combined the information on the period and the damping rate. In that case, we added a new observable, but also a new free parameter: the internal Alfvén speed (or travel time). In principle this may not seem to constitute a particular gain, but after obtaining the $1 \mathrm{D}$ solution curve in the three-dimensional parameter space, the projection of the solution curve onto the Alfvén speed axis turns out to be in a rather limited interval. As we still have a 1D solution curve, the observed values of period and damping time of the fundamental kink oscillation mode do not allow a unique identification of even a 1D equilibrium model, unless some additional assumption is made (like in the analysis of Goossens et al. 2002, by assuming a value for the density contrast). However, even without additional information, we can determine a rather constrained range for the internal Alfvén speed.

Acknowledgements. The authors acknowledge Ronald Van der Linden, who originally developed the LEDA code, as well as all others who perfected them.
This research was funded by K.U. Leuven project GOA/2004/01, FWO project G.0451.05 and PRODEX project C90203. The numerical results were obtained on the HPC cluster VIC (K.U. Leuven).

\section{References}

Andries, J., Arregui, I., \& Goossens, M. 2005a, ApJ, 624, L57

Andries, J., Goossens, M., Hollweg, J. V., Arregui, I., \& Van Doorsselaere, T. 2005b, A\&A, 430, 1109

Arregui, I., Van Doorsselaere, T., Andries, J., Goossens, M., \& Kimpe, D. 2005, A\&A, 441, 361

Aschwanden, M. J., Fletcher, L., Schrijver, C. J., \& Alexander, D. 1999, ApJ, 520,880

Aschwanden, M. J., De Pontieu, B., Schrijver, C. J., \& Title, A. M. 2002, Sol. Phys., 206, 99

Aschwanden, M. J., Nightingale, R. W., Andries, J., Goossens, M., \& Van Doorsselaere, T. 2003, ApJ, 598, 1375

De Pontieu, B., Martens, P. C. H., \& Hudson, H. S. 2001, ApJ, 558, 859

Goossens, M., Hollweg, J. V., \& Sakurai, T. 1992, Sol. Phys., 138, 233

Goossens, M., Andries, J., \& Aschwanden, M. J. 2002, A\&A, 394, L39

Goossens, M., Andries, J., \& Arregui, I. 2006, Roy. Soc. London Philos. Trans. Ser. A, 364, 433

Heyvaerts, J., \& Priest, E. R. 1983, A\&A, 117, 220

Hollweg, J. V., \& Yang, G. 1988, J. Geophys. Res., 93, 5423

Nakariakov, V. M., \& Ofman, L. 2001, A\&A, 372, L53

Nakariakov, V. M., Ofman, L., DeLuca, E. E., Roberts, B., \& Davila, J. M. 1999, Science, 285,862

Ofman, L. 2002, ApJ, 568, L135

Ofman, L., \& Aschwanden, M. J. 2002, ApJ, 576, L153

Roberts, B. 2000, Sol. Phys., 193, 139

Roberts, B., Edwin, P. M., \& Benz, A. O. 1984, ApJ, 279, 857

Ruderman, M. S. 2005, in The Dynamic Sun: Challenges for Theory and Observations, ESA SP-600

Ruderman, M. S., \& Roberts, B. 2002, ApJ, 577, 475

Schrijver, C. J., \& Brown, D. S. 2000, ApJ, 537, L69

Schrijver, C. J., Aschwanden, M. J., \& Title, A. M. 2002, Sol. Phys., 206, 69

Tirry, W. J., \& Goossens, M. 1996, ApJ, 471, 501

Uchida, Y. 1970, PASJ, 22, 341

Van der Linden, R. A. M. 1991, Ph.D. Thesis, K. U. Leuven

Van Doorsselaere, T., Andries, J., Poedts, S., \& Goossens, M. 2004, ApJ, 606, 1223

Verwichte, E., Nakariakov, V. M., Ofman, L., \& Deluca, E. E. 2004, Sol. Phys., 223, 77

Verwichte, E., Foullon, C., \& Nakariakov, V. M. 2006, A\&A, 452, 615 